\title{
Impact of Wind Generation Variability on Small Signal Stability of Power Systems
}

\author{
Mehdi Garmroodi ${ }^{1}$, Student Member, IEEE, David J. Hill ${ }^{1,2}$, Fellow, IEEE, Jin Ma ${ }^{1}$, Member, IEEE \\ and Gregor Verbič ${ }^{1}$, Senior Member, IEEE \\ ${ }^{1}$ School of Electrical and Information Engineering, The University of Sydney, Sydney, New South Wales, Australia \\ ${ }^{2}$ Department of Electrical and Electronic Engineering, The University of Hong Kong, Hong Kong \\ Emails:\{mehdi.garmroodi, david.hill, j.ma, gregor.verbic\}@sydney.edu.au
}

\begin{abstract}
Power systems are shifting toward utilizing higher penetration of renewable energy sources (RESs) and especially wind generation because of the need to reduce carbon emissions over the next several decades. Small signal stability can be influenced with increasing penetration of wind generation in power systems. Poor damping of low frequency electromechanical oscillations are a threat to secure operation of power systems. In this paper, we investigate the sensitivity of the small signal stability of a two area test system on wind generation variability, mainly focusing on the stability of inter-area oscillation mode. Margins that determine the minimum amount of wind generation for a stable system for different tie-line power values are derived. Static load models are used and the impact of different flexibility levels from loads on the stability margins are studied. Furthermore, the impact of the proximity of flexible load to wind generation is also studied.
\end{abstract}

Keywords-Inter-area oscillation mode, modal analysis, small signal stability, static load model, tie-power, wind generation

\section{INTRODUCTION}

Concerns about global warming and climate change have necessitated aggressive reduction in greenhouse gas emissions over the next several decades. The power sector with $26 \%$ share of global greenhouse gas emissions is a major contributor of emissions [1]. As progressive actions, governments are using both mandatory targets and/or incentive schemes to push utilities toward providing their energy from renewable energy sources (RESs). Among different RESs, wind turbine generators (WTGs) have gained considerable attention because of their mature technology and attractive economics [2], [3].

Traditionally, conventional synchronous generators were the main components providing synchronizing and damping torque to the system to damp out power system oscillations and mitigate instability. Increased penetration of wind energy could affect operation and security of power systems in different ways. These resources are normally connected to the grid with a power electronic interface and they do not introduce inertia to the system. Once they displace some conventional generation, system inertia will be reduced. Moreover, dynamics of WTGs are inherently different from synchronous generators and even though they do not introduce new oscillatory modes to the system [4], [5], they might alter some of the existing system modes with changing power flow paths and by interacting with synchronous generators.

Several research efforts have been devoted to address the impact of wind generation on system damping [6]-[9]. Some references have reported improvements in system damping [8]-[12], while others have observed some deteriorations [4], [6]. In [4] damping performance of the Queensland network is studied assuming different scenarios including load growth and synchronous generation displacement to accommodate wind generation. Deteriorations in system damping are observed in some scenarios while improvements are observed in some others. However, the variability and intermittency on wind generation is not considered in that study and it is assumed that WTGs are operating at their nominal power. A study by NEMMCO [6] evaluated the effect of increased penetration of wind energy in South Australia on the stability of NEM and it has reported some reductions in system damping in some cases. A review paper on the impact of wind energy on power system oscillations reveals that the influence of variable speed wind turbines on system oscillations does not draw a clear answer and is affected by many factors like wind turbine technology, control mode and wind penetration [5]. Some papers propose tools to assess the impact of WTGs on system stability. For instance, replacing WTGs with an equivalent synchronous generator and sensitivity to inertia is used in [7], [13], [14] to evaluate the impact of RESs on system stability. In [7] a sensitivity analysis is carried out to evaluate the effect of doubly fed induction generator (DFIG) based WTGs on small signal stability of a power system. WTGs are substituted with conventional synchronous generators and the sensitivity of system modes to inertia is used as a measure for improvement or deterioration in system damping.

Most of the recent studies have looked at the outcomes of stability studies for a specific network under a specific situation without offering any universal guidelines. In fact, there are many uncertainties related to RESs that demands a sensitivity analysis to evaluate their effects on power system stability. In this paper, we have focused on the impact of variability in the output power of wind generators on the small signal stability of system. A well-known two area test network [15] is used for the analysis where wind generation displaces one of the synchronous generators in the network. Margins are obtained that determine minimum wind generation to maintain system stability and the sensitivity of these margins to load flexibility and location of flexible load is studied.

The rest of the paper is organized as follows. Small signal stability is described in section II. The test system and load modelling used throughout the study in presented in section III. The impact of wind generation variability on small signal stability is demonstrated in Section IV and in Section V the 
impact of load flexibility and location of flexible load on inter-area oscillation mode is studied. Finally, conclusions are presented in Section V.

\section{Small Signal Stability}

Power systems are nonlinear dynamic systems that can be described by differential equations of the form:

$$
\begin{array}{r}
\dot{\mathbf{x}}=\mathbf{f}(\mathbf{x}, \mathbf{u}) \\
\mathbf{y}=\mathbf{g}(\mathbf{x}, \mathbf{u})
\end{array}
$$

where, $\mathbf{x}=\left[x_{1}, x_{2}, \ldots, x_{n}\right]^{T}$ is the vector of state variables, $\mathbf{u}=\left[u_{1}, x_{2}, \ldots, x_{m}\right]^{T}$ is the vector of system inputs and $\mathbf{y}=$ $\left[y_{1}, y_{2}, \ldots, y_{p}\right]^{T}$ is the vector of system outputs. Small signal stability of the system is analyzed by linearizing equation (1) around the operating point to give the form:

$$
\begin{aligned}
& \dot{\Delta} \mathrm{x}=\mathrm{A} \Delta \mathrm{x}+\mathrm{B} \boldsymbol{\Delta} \mathrm{u} \\
& \Delta \mathrm{y}=\mathrm{C} \Delta \mathrm{x}+\mathrm{D} \boldsymbol{\Delta} \mathrm{u}
\end{aligned}
$$

where, $\mathbf{A} \in \mathbb{R}^{n \times n}$ is the state matrix, $\mathbf{B} \in \mathbb{R}^{n \times m}$ is the input matrix, $\mathbf{C} \in \mathbb{R}^{p \times n}$ is the output matrix and $\mathbf{D} \in \mathbb{R}^{p \times m}$ defines the proportion of input which directly appears in the output.

Judgment about the system stability can be done by investigating the loci of eigenvalues of $\mathbf{A}$. Each eigenvalue can be expressed as:

$$
\lambda_{i}=\sigma_{i} \pm j \omega_{i}
$$

If all the eigenvalues have negative real parts, system (2) is asymptotically stable and, by Lyapunov's first method, so is the system (1) in a local sense. An (underdamped) oscillatory response occurs when at least one eigenvalue pair has $\omega_{i} \neq 0$. If all the eigenvalues have $\omega_{i}=0$ then the response is nonoscillatory. For the special situation when an eigenvalue pair cross the $j \omega$ axis, the system becomes unstable, but we need to refer to the theory of oscillations in nonlinear systems [16] and use the nonlinear model (1) to infer the exact behaviour at the point of crossing. $\sigma_{i}$ determines the rate of damping in a specific mode and damping ratio is defined by:

$$
\zeta_{i}=-\sigma_{i} / \sqrt{\sigma_{i}^{2}+\omega_{i}^{2}}
$$

Power grids experience some different types of oscillatory modes after a small disturbance occurs in the system [15]. For simplicity, we will regard a Local oscillation as between one generator and the rest of the system. On the other hand, in Inter-area oscillations, a group of generators in one area oscillate against another group of generators which are typically electrically distant. Electromechanical oscillations in the system happen in the frequency range of 0.1 to $3 \mathrm{~Hz}$ and normally are the lowest damped modes in the system. Interarea modes generally occur across heavily loaded or weak transmission paths at the frequencies between $0.1 \mathrm{~Hz}$ and 1 $\mathrm{Hz}[17]$.

\section{Test System AND LOAD MOdELling}

\section{A. System Description}

Fig. 1 shows single line diagram of the test system used in this study. Test system is comprised of four generators and two load centers which are grouped in two areas. These two

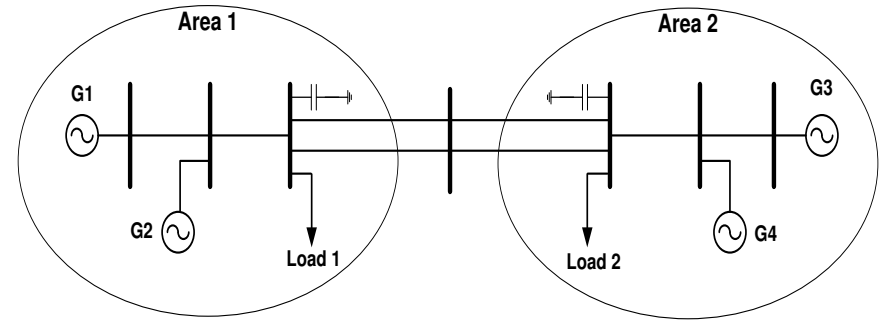

Fig. 1. Test System

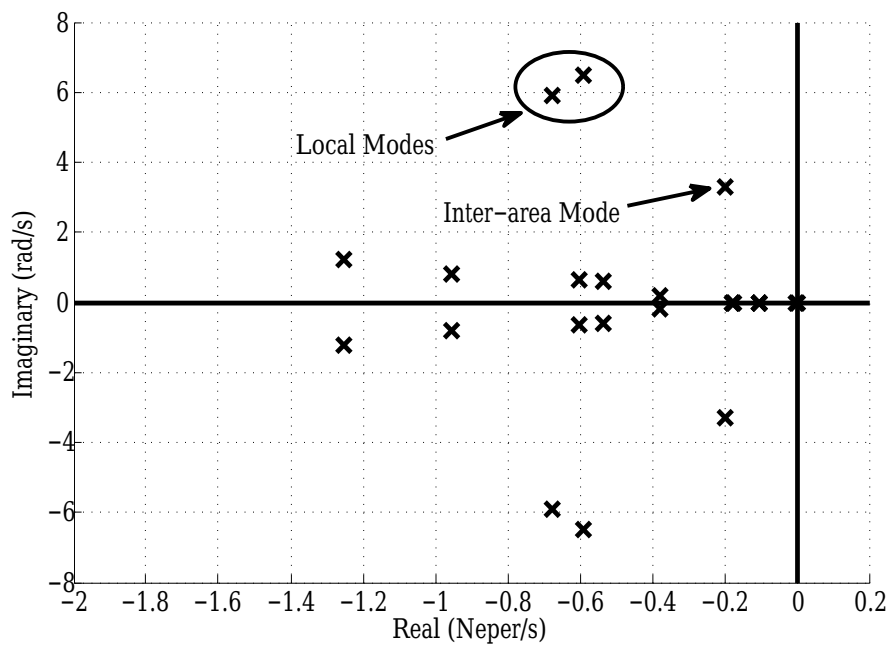

Fig. 2. Eigenvalue plot of the base case system

area are connected together with two long transmission lines. All generators are equipped with AVR and PSS adopted from [15]. Generator G1 in area 1 is considered as slack machine and the other generators are each supplying $700 \mathrm{MW}$ of active power to the system. Total demand in the system is $2500 \mathrm{MW}$ and sharing this demand between two loads results in different values for tie power. Load buses at area 1 and area 2 are compensated with 100 MVAR and 250 MVAR shunt capacitor banks respectively. Modal analysis of this test system is shown in Fig. 2 and shows two local and one inter-area oscillation modes. Local oscillation modes correspond to generators G1 and G2 in the area 1 and generators G3 and G4 in the area 2. Due to the long transmission line between area 1 and area 2 , there exist an inter-area mode in which generators G1 and G2 in area 1 oscillate against generators G3 and G4 in area 2. This mode is the lowest damped mode in the system. In Fig. 1, all generators are represented as conventional synchronous generations and it will referred to as base case. Then, generator G2 is replaced with a fully rated converter WTG to capture the effects of replacing conventional generation with WTG. The test system is modeled in DIgSILENT Power Factory 15.1 and a generic model of wind turbine with all the controllers is used.

\section{B. Load Modelling}

Understanding correct behavior of power systems requires proper modelling of system devices and components. Among different system components, the importance of proper representation of loads in power system stability studies has long 
TABLE I. EXPONENTS $\alpha$ AND $\beta$ FOR SOME TYPICAL LOADS

\begin{tabular}{|c|c|c|}
\hline Load & $\alpha$ & $\beta$ \\
\hline Constant Power & 0 & 0 \\
\hline Constant Current & 1 & 1 \\
\hline Constant Impedance & 2 & 2 \\
\hline Air-Conditioner & 0.088 & 2.5 \\
\hline Refrigerator & 0.77 & 2.5 \\
\hline Incandecent Lights & 1.55 & 0 \\
\hline
\end{tabular}

been recognized [18], [19]. Load modelling in high voltage systems is a relatively difficult task due to the various devices involved. Traditionally, load models are classified into static and dynamic load models. In static load models, which are considered here, active and reactive power of loads are voltage dependent and are expressed as follows:

$$
\begin{gathered}
P=P_{0}\left(\frac{V}{V_{0}}\right)^{\alpha} \\
Q=Q_{0}\left(\frac{V}{V_{0}}\right)^{\beta}
\end{gathered}
$$

where, $P$ and $Q$ are the active and reactive power of loads at voltage $V ; P_{0}, Q_{0}$ and $V_{0}$ are steady state active, reactive and voltage of loads. Exponents $\alpha$ and $\beta$ determine the load characteristic and for typical loads, these values are in the range of $0<\alpha<3$ and $0<\beta<7$ [15], [19]. Values of $\alpha$ and $\beta$ for some typical loads are presented in Table I.

\section{IMPACT OF WIND GENERATION ON INTER-AREA OSCILLATION MODE}

\section{A. Fixed Power from Wind Generation}

Increased penetration of wind generation can be accommodated in different ways using load response and/or dispatch of conventional generation. Here, it is assumed that generator G2 is replaced with a fully rated converter WTG and it delivers the same amount of power the grid as the G2 did. Modal analysis is used to study the impact of increased penetration of wind on damping of inter-area mode. Due to a discrete change in the network (replacing G2 with WTG), system dynamics are different and this can be shown in the number of system eigenvalues where some of the system modes disappear and some new modes appear. One of the main changes in system modes is that local area oscillation mode of area 1 will disappear because the WTG does not introduce inertia to the system. However, the inter-area mode will remain in place because of the rotating inertia of synchronous generators in both areas. Damping of the inter-area mode for base case and wind generation case in different tie-power values is compared in Table II. As it is seen from the table, system damping is decreased with decreasing tie-line power in the base case. The impact of incorporating wind power on damping of inter-area mode is that it deteriorates system damping in high tie-power values and improves it in low tie-power flows. Moreover, comparing the base case and the wind generation case show that the damping ratio of the inter-area mode is less sensitive to tie-power in the wind generation case. However, the system has better damping performance in case of high tie-power flows.

\section{B. Variable Power from Wind Generation}

In most of the recent studies on evaluating the impact of wind generation on system damping, variability of wind gen-
TABLE II. DAMPING OF INTER-AREA Mode IN DiFFERENT TIE-POWER VAlues

\begin{tabular}{|c|c|c|c|c|}
\hline \multirow{2}{*}{$\begin{array}{c}\text { Tie-Line } \\
\text { Power }\end{array}$} & \multicolumn{2}{|c|}{ Base Case } & \multicolumn{2}{c|}{ WTG Case } \\
\cline { 2 - 5 } & $\begin{array}{c}\text { Damping } \\
\text { Ratio (\%) }\end{array}$ & $\begin{array}{c}\text { Damped } \\
\text { Freq. (Hz) }\end{array}$ & $\begin{array}{c}\text { Damping } \\
\text { Ratio (\%) }\end{array}$ & $\begin{array}{c}\text { Damped } \\
\text { Freq. (Hz) }\end{array}$ \\
\hline 460 MW & 6.1 & 0.56 & 5.5 & 0.55 \\
\hline 300 MW & 5.3 & 0.56 & 5.2 & 0.56 \\
\hline 150 MW & 4.6 & 0.56 & 5.1 & 0.57 \\
\hline 30 MW & 3.9 & 0.55 & 5.0 & 0.56 \\
\hline
\end{tabular}

eration is neglected and it is assumed that WTGs are operating at their maximum capacity. Considering the intermittent nature of wind power, load-frequency control systems should have the ability to cope with this intermittency and provide enough power to balance system generation and demand. However, small signal stability of the system will also be affected with the variability of wind generation. For example, Fig. 3 depicts root-locus of system eigenvalues when output power of WTG in the test system is decreased from its nominal value to a minimum of $75 \mathrm{MW}$. As it is seen, when the WTG is operating at its full capacity, the inter-area oscillation mode is located in the Left Half Plane (LHP) and the system is stable. Decreasing power from the WTG moves this mode toward the Right Half Plane (RHP) and the system becomes unstable. The result of modal analysis in Fig. 3 also shows the local area mode corresponding to area 1 has disappeared and the local mode of area 2 does not get affected when the output power of the WTG varies. This is because the power of the synchronous generators in area 2 remains constant and they do not engage in system balancing. Therefore, in a realistic judgment about the impact of wind generation on system stability, intermittency of these resources should also be considered.

For comparison purposes, similar variation is applied to the output power of synchronous generator G2 in the base case and root-locus of eigenvalues are shown in Fig. 4. Similar to the wind generation case, the inter-area mode is moving toward the RHP and its damping is decreased. However, at the minimum generation of $\mathrm{G} 2$, this mode does not leave the LHP and the system remains stable. This is because of the fact that synchronous generators are able to provide synchronizing and damping torque to the system; something that WTGs are unable to. In the wind generation case, reduced power from the WTG should be supplied by generator G1 which is the slack generator. Providing more power to the grid, generator G1 should operate at higher internal angle value and therefore its ability to provide synchronizing torque to the system decreases. In the base case, however, decreasing output power of generator G2 increases its ability to support the stability with synchronizing torque due to its lower internal angle. Thus, the inter-area mode does not leave the LHP and small signal stability is maintained for the whole range of power from G2. Regarding local area modes, the damping of the mode corresponding to area 1 is increased and then it has decreased. However, the local mode of area 2 is remained unchanged due to the same reason in wind generation case.

Fig. 3 illustrates that below a specific amount of wind generation, the eigenvalue corresponding to inter-area mode crosses the imaginary axis and enters the RHP. Thus, a minimum amount of wind generation is required to maintain system stability. Simulation results show that the amount of this minimum wind power depends on system characteristics (like inertia, load characteristics, etc.) as well as the amount 


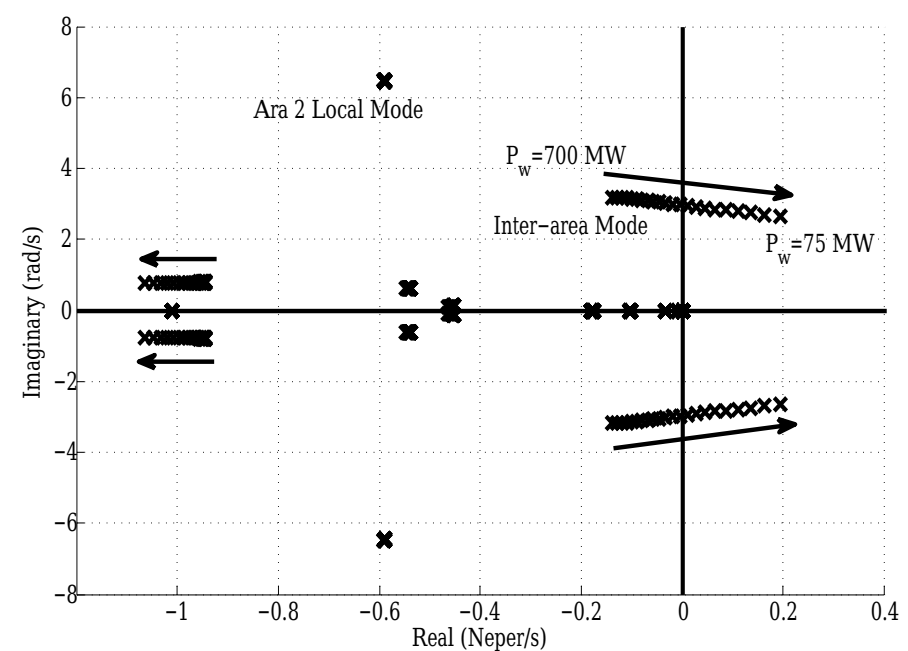

Fig. 3. Inter-area mode for decreasing wind power from $700 \mathrm{MW}$ to $75 \mathrm{MW}$

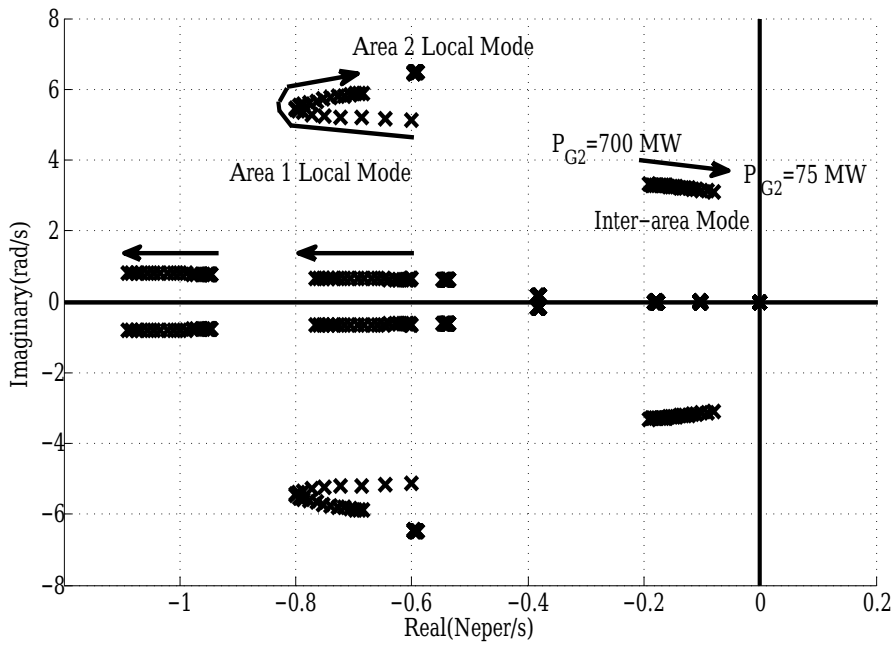

Fig. 4. Dominant eigenvalues when output power of $\mathrm{G} 2$ is decreased from $700 \mathrm{MW}$ to $75 \mathrm{MW}$

of tie power. In other words, the level of wind penetration below which the inter-area mode crosses the imaginary axis and enters RHP is dependent on the amount of tie power. This value for different tie-power values are calculated and shown in Fig. 5. This figure shows a margin that determines the lowest wind power required to keep the system stable. In this figure, the vertical axis is the penetration of wind power in area 1 calculated from:

$$
P_{\text {wind }}(\%)=\frac{P_{\text {wind }}}{P_{\text {wind }}+P_{G 1}}
$$

The area above the graph is called Stable Region meaning that the system is stable for a specific tie-power if wind penetration in area 1 is above the margin. On the other hand, in the Unstable Region, the inter-area mode is placed in RHP and system is unstable. Fig. 5 shows that the minimum wind penetration required for a stable system increases with the amount of tie-power. Load characteristic influences this margin and this is discussed in the following section.

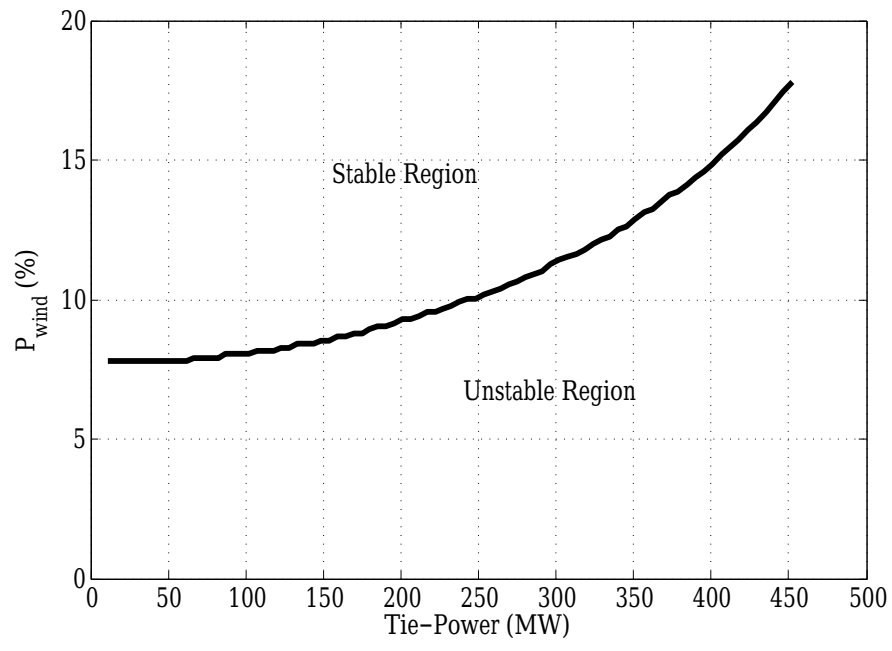

Fig. 5. Stability margin determining minimum wind generation required for a stable system

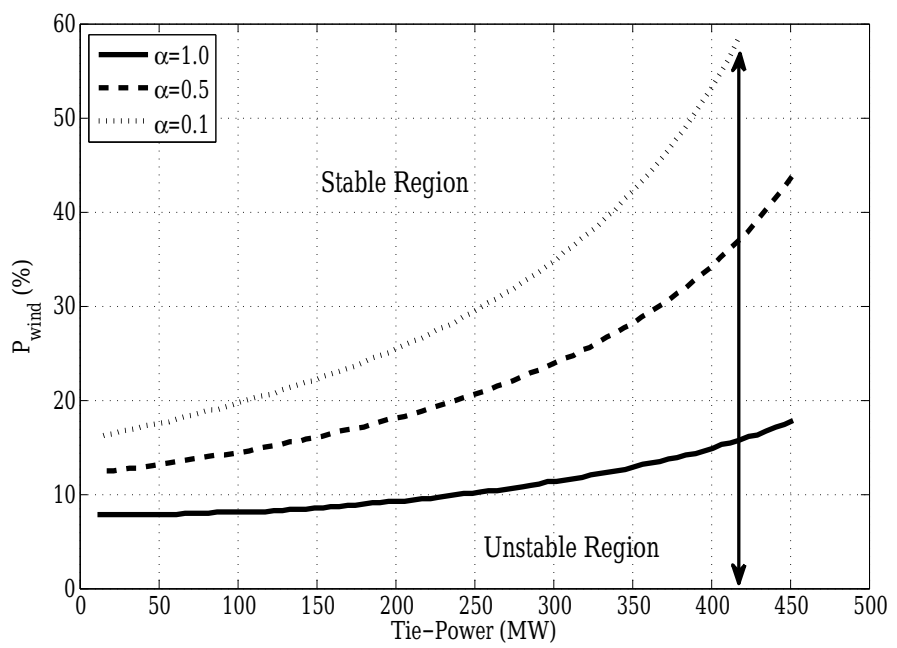

Fig. 6. Impact of load flexibility on stability margin

\section{IMPACT OF LOAD FLEXIBILITY AND LOCATION}

In the static load model discussed in section III, exponents $\alpha$ and $\beta$ reflect the flexibility of loads to a change in bus voltage. Higher values of $\alpha$ and $\beta$ result in larger variations in the active and reactive power demand of loads, representing more flexibility from loads to system conditions. Fig. 6 shows the impact of different flexibility levels from loads on the stability margin. As it is seen, when the flexibility of loads decrease and they are getting closer to constant power loads, the stability margin is located higher in the "Tie-power$P_{\text {wind }}$ " plane and the stable region is smaller. This implies that for a specific tie-power, the amount of wind generation required for a stable system increases for lower values of $\alpha$. The difference between stability margins are more significant for high tie-power values where the graphs are further apart. This is more crucial when $\alpha=0.1$ and the system is unstable above 420MW of tie-power even for the maximum wind power generated by WTG (700 MW).

Fig. 6 illustrates that higher flexibility from loads improves system stability. However, the level of impact from load 1 and 


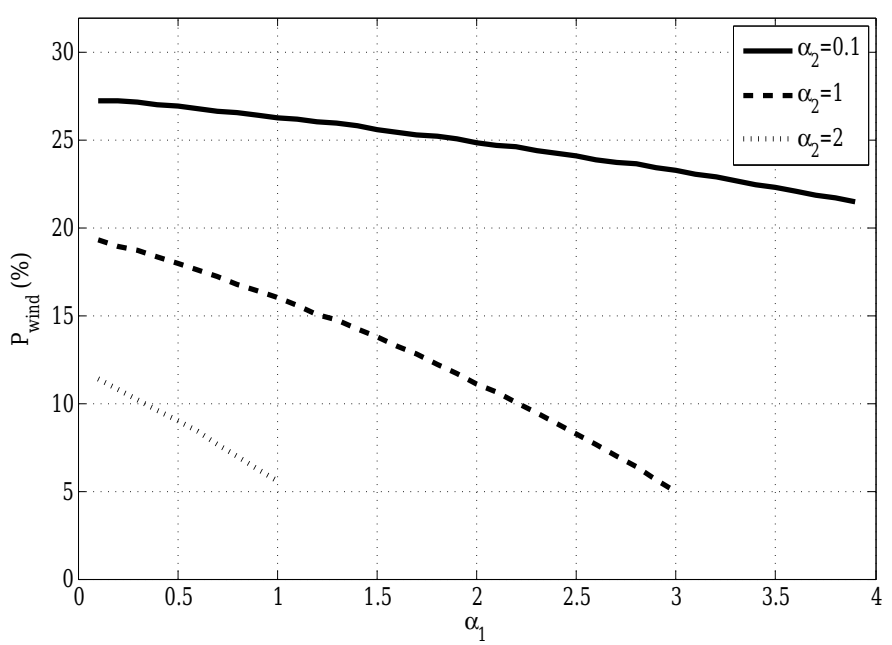

Fig. 7. Impact of flexibility of Load 1 on stability of inter-area mode

load 2 on stability margin is different. Load 1 is located in area 1 where there is lower kinetic energy from rotational mass and part of power is generated by WTG. In area 2, however, all the power is generated by conventional synchronous generation. In order to analyze the impact of location of the flexible load on stability of inter-area mode, a similar demand (1100 MW) for load 1 and load 2 is considered and stability margins are derived for different $\alpha_{1}$ and $\alpha_{2}$ values. In Fig. 7 (Fig. 8) stability margins for different values of $\alpha_{2}\left(\alpha_{1}\right)$ where $\alpha_{1}$ $\left(\alpha_{2}\right)$ is swept between 0 and 4 is shown. Note that the stability margins show the minimum wind penetration required for a stable system in a specific tie-power (118 MW) and different values of $\alpha_{1}$ and $\alpha_{2}$. It is seen in Fig. 7 and Fig. 8 that increasing flexibility from both loads improves system stability. However, the level of impact from load 1 and load 2 is different. A comparison between the two figures shows that flexibility from Load 2 is more influential on the stability of inter-area mode. In Fig. 7 there is an almost flat stability margin when $\alpha_{2}$ is 0.1 and $\alpha_{1}$ is swept between 0 and 4 . This demonstrates that in this case flexibility of load 1 does not improve system stability considerably. On the other hand in Fig. 8, the stability margins decrease more sharply with increasing of $\alpha_{2}$ showing more impact from flexibility of load 2 on stability of inter-area mode.

\section{CONClusion}

In this paper, the impact of variable power from wind generators on the small signal stability of a system is studied. A well-known four generator, two area system is used as a test bed and it is assumed that WTG displaces one of the synchronous generators in the grid. Modal analysis is carried out for different levels of output power from WTG and system modes are tracked.

Simulation results show that displacement of conventional generation with WTG might be a threat to small signal stability of the system, especially in low wind penetration levels. Moreover, inter-area power flow and load flexibility are two factors affecting the minimum wind generation required for a stable system. In the former one, increased tie-power deteriorates small signal stability by increasing the minimum level of wind

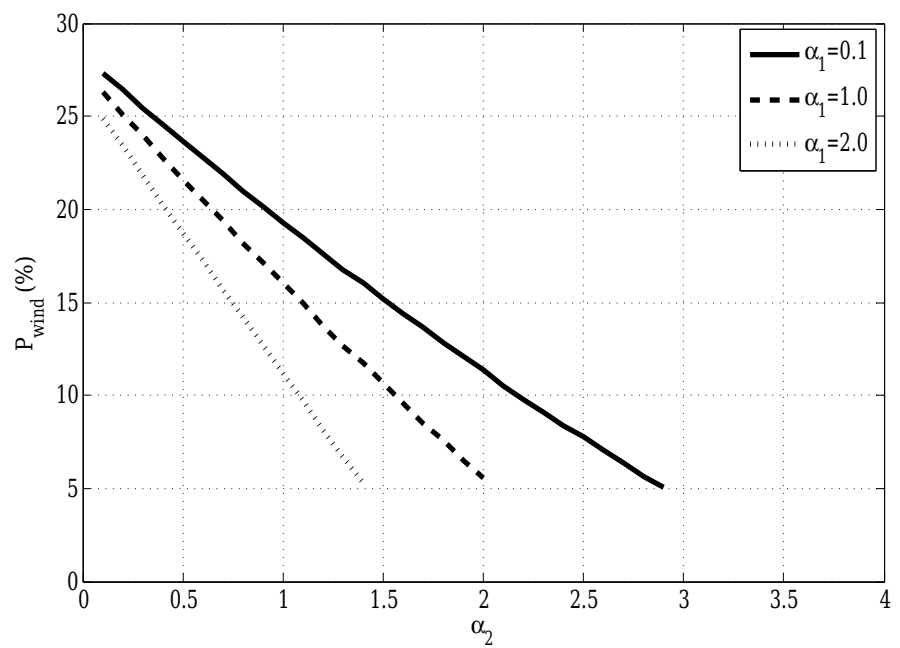

Fig. 8. Impact of flexibility of Load 2 on stability of inter-area mode

penetration needed in the system. In the latter one, increased flexibility from loads improves system stability and a system with more flexible loads are less prone to instability.

\section{REFERENCES}

[1] United States Environmental Protection Agency, "Global greenhouse gas emissions data." [Online]. Available: http://www.epa.gov/climatechange/ghgemissions/global.html

[2] P. Pourbeik, R. Koessler, D. Dickmander, and W. Wong, "Integration of large wind farms into utility grids (part 2 - performance issues)," in Power Engineering Society General Meeting, 2003, IEEE, vol. 3, July 2003, pp. -1525 Vol. 3.

[3] L. Meegahapola and D. Flynn, "Impact on transient and frequency stability for a power system at very high wind penetration," in Power and Energy Society General Meeting, 2010 IEEE, July 2010, pp. 1-8.

[4] N. Modi, T. K. Saha, and T. Anderson, "Damping performance of the large scale queensland transmission network with significant wind penetration," Applied Energy, vol. 111, no. 0, pp. $225-233,2013$. [Online]. Available: http://www.sciencedirect.com/science/article/pii/S0306261913004145

[5] J. L. Domnguez-Garca, O. Gomis-Bellmunt, F. D. Bianchi, and A. Sumper, "Power oscillation damping supported by wind power: A review," Renewable and Sustainable Energy Reviews, vol. 16, no. 7, pp. 4994 - 5006, 2012. [Online]. Available: http://www.sciencedirect.com/science/article/pii/S136403211200247X

[6] H. M. M. Poller, K. Theron, P. Ravalli, and R. A., "Assessment of potential security risks due to high levels of wind generation in south australia-summary of digsilent studies," National Electricity Market Management Company Limited (NEMMCO), Tech. Rep., 2005.

[7] D. Gautam, V. Vittal, and T. Harbour, "Impact of increased penetration of dfig-based wind turbine generators on transient and small signal stability of power systems," Power Systems, IEEE Transactions on, vol. 24, no. 3, pp. 1426-1434, Aug 2009.

[8] E. Vittal and A. Keane, "Rotor angle stability with high penetrations of wind generation," in Power and Energy Society General Meeting, 2012 IEEE, July 2012, pp. 1-1.

[9] L. Meegahapola, D. Flynn, and T. Littler, "Transient stability analysis of a power system with high wind penetration," in Universities Power Engineering Conference, 2008. UPEC 2008. 43rd International, Sept 2008, pp. 1-5.

[10] E. Muljadi, C. Butterfield, B. Parsons, and A. Ellis, "Effect of variable speed wind turbine generator on stability of a weak grid," Energy Conversion, IEEE Transactions on, vol. 22, no. 1, pp. 29-36, March 2007. 
[11] R. H. Byrne, R. T. Elliott, J. C. Neely, C. A. Silva-Monroy, D. A. Schoenwald, and L. L. Grant, "Renewable source controls for grid stability," Sandia National Laboratories, Tech. Rep., 2012.

[12] G. Tsourakis, B. Nomikos, and C. Vournas, "Contribution of doubly fed wind generators to oscillation damping," Energy Conversion, IEEE Transactions on, vol. 24, no. 3, pp. 783-791, Sept 2009.

[13] S. Eftekharnejad, V. Vittal, G. Heydt, B. Keel, and J. Loehr, "Impact of increased penetration of photovoltaic generation on power systems," Power Systems, IEEE Transactions on, vol. 28, no. 2, pp. 893-901, May 2013.

[14] _ "Small signal stability assessment of power systems with increased penetration of photovoltaic generation: A case study," Sustainable Energy, IEEE Transactions on, vol. 4, no. 4, pp. 960-967, Oct 2013.

[15] P. Kundur, Power System Stability and Control. McGraw-Hill Education, 1994.

[16] J. Hale and H. Kocak, Dynamics and Bifurcations. Springer, 1991.

[17] C. Silva-Monroy, J. Neely, R. Byrne, R. Elliott, and D. Schoenwald, "Wind generation controls for damping of inter-area oscillations," in Power and Energy Society General Meeting (PES), 2013 IEEE, July 2013, pp. 1-5.

[18] C. Concordia and S. Ihara, "Load representation in power system stability studies," Power Apparatus and Systems, IEEE Transactions on, vol. PAS-101, no. 4, pp. 969-977, April 1982.

[19] I. Hiskens and J. Milanovic, "Load modelling in studies of power system damping," Power Systems, IEEE Transactions on, vol. 10, no. 4, pp. 1781-1788, Nov 1995 